\title{
Association Between Waist Circumference Change and Incident Chronic Obstructive Pulmonary Disease in Chinese Adults: An 18-year Cohort Study
}

\section{Yun-Lei Ma}

Hebei Medical University College of Traditional Chinese Medicine

Han-Jun Zhao

Hebei Medical University Fourth Affiliated Hospital and Hebei Provincial Tumor Hospital

Ying-Hao Su ( $\sim$ suyinghao2020@163.com )

Department of Respiratory Medicine, Traditional Chinese Medicine Hospital of Hebei Province, 389

Zhongshan Road, Shijiazhuang 050017, China https://orcid.org/0000-0002-9929-244X

\section{Research}

Keywords: waist circumference, abdominal obesity, chronic obstructive pulmonary disease, Chinese adults

Posted Date: August 31st, 2020

DOI: https://doi.org/10.21203/rs.3.rs-66864/v1

License: (a) (i) This work is licensed under a Creative Commons Attribution 4.0 International License. Read Full License 


\section{Abstract}

Background The aim of our study was to investigate waist circumference (WC) change and the risk of incident chronic obstructive pulmonary disease (COPD) among Chinese adults.

Methods A total of 8,164 participants aged 20-35 years were recruited who attended health examination with the repeat measurements of WC and lung function (forced vital capacity (FVC), forced expiratory volume in $\left.1 \mathrm{~s}\left(\mathrm{FEV}_{1}\right)\right)$ from 2001 to 2019. The data of same measurements were collected at their age of 3853 years. Participants were categorized by WC change as $\leq-2.5 \%,-2.5 \%$ to $2.5 \%, 2.5 \%$ to $5 \%$ and $₫ 5 \%$ according to gender. The modified Poisson regression models were used to assess the association of WC gain and the risk of COPD.

Results During 18-year follow-up, a total of 917 COPD cases were identified. From baseline to follow-up, the mean of $\mathrm{FEV}_{1}$ decreased from $3.20 \mathrm{~L}$ to $2.79 \mathrm{~L}$ among male participants and $2.28 \mathrm{~L}$ to $1.95 \mathrm{~L}$ among female participants. Comparing with participants who were not abdominal obesity at both baseline and follow-up, participants with abdominal obesity among both genders after the follow-up were associated to the greater risk of COPD regardless of abdominal obesity at baseline. The risk of incident COPD increased 19\% among male participants $(R R=1.19,95 \% C l=1.04-1.48)$ and $14 \%$ among female participants $(R R=1.14,95 \% C l=1.01$ 1.40) when WC gain increased $\triangle 5 \%$ during the 18 -year follow-up. The COPD risk decreased $28 \%$ among male participants with WC change $\leq-2.5 \%(R R=0.82,95 \% C l=0.67-0.99)$.

Conclusions The risk of incident COPD was positively associated with increasing WC for both genders among Chinese adults.

\section{Background}

The morbidity and mortality of chronic obstructive pulmonary disease (COPD) is increasing dramatically in past decades around the world.[1] World Health Organization (WHO) predicted that the mortality of COPD will rank from the fourth leading cause of death in 2004 to the third by 2030, with more than $90 \%$ of the morbidity and mortality of COPD occurring in low- and middle-income countries.[2-3] As shown in a recent meta-analysis in Chinese, the age-adjusted prevalence of COPD defined by spirometry test increased rapidly from $8.2 \%$ in $2002-2004$ to $13.7 \%$ in $2012-2015$.[4]

Available studies suggested that several factors were associated with increasing risk of COPD including higher BMI (body mass index), increasing age, tobacco smoking, household air pollution, and occupational exposures.[5-9] The association of general obesity $\left(\mathrm{BMI} \geq 30 \mathrm{~kg} / \mathrm{m}^{2}\right)$ and the risk of COPD received much attention for long time, but there were no consistent conclusions at present. On the one hand, some studies suggested that BMI was inversely associated with the risk of COPD.[9-11] On the other hand, the "Obesity Paradox" of COPD existed in many studies showed that overweight/obese was helpful to improved COPD patients' survival.[12-13] However, the common definition for obesity or adiposity relied on BMI, which is simple and reasonable. WC as another measurement of obesity was better to reflect the distribution of fat tissue throughout the body. However, there were a lack of studies regarding to the association of WC with lung function, and available studies only conducted in children and adolescence showed complex results. 


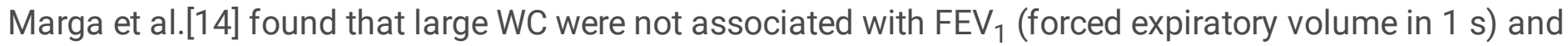
FVC (forced vital capacity) in children aged 8 years old, while Feng et al.[15] suggested that WC was inversely associated with lung function in Chinese children and adolescence.

Thus, our study aimed to assess the association of lung function and the risk of incident COPD, with 18-year changes in adiposity as measured by WC in Chinese adults. Furthermore, this relationship was investigated by gender.

\section{Materials And Methods Study population}

This longitude cohort consisted of 11,357 participants residing in Shijiazhuang city which is the provincial capital city of Hebei province in China. All participants have attended the health examinations conducted in 21 public medical institutions during 2000-2019. The survey was initially conducted during March to April 2000 to March to April 2001 for participants aged over 18 years old. But participants with missing data including age, WC, $\mathrm{FVE}_{1}$ and FVC ( $\left.\mathrm{n}=2,589\right)$, as well as pregnant and breastfeeding participants $(n=157)$ were excluded. Additionally, participants were excluded with underlying lung disease: asthma and asthmalike symptoms including wheeze, nocturnal chest tightness, attacks of breathlessness after activity or at rest or at night time, use of inhaled or oral medicine for breathing problems $(n=392)$. Considering possible confounding by disease- and metabolic WC change, we also excluded participants who had diabetes ( $\mathrm{n}=$ $52)$ and cancer $(n=3)$. Finally, our study included 8,164 participants $(3,929$ men and 4,235 women) to estimate the risk of incident COPD with change in WC during the 18-year follow-up.

Ethical approval was obtained from the ethics committees of all participating institutions and all participants provided informed written consent.

\section{Data Collection}

Before the health examination, all participants need to finish an overnight fast. At baseline, the questionnaires were used to collect the information of social and demographic factors including age, gender, educational level, smoking habits, alcohol consumption and physical activities. The same questionnaires were applied for the information collection in each year. Educational level was categorized as low level (lower than senior high school) and high level (college or university degree and master's degree or higher). Smoking status was categorized as currently smoking, ever smoking or never smoking. Alcohol drinking was categorized as drinker (alcohol consumption 12 or more timed in the last year) and non-drinker (alcohol consumption less than 12 times in the last year). Urbanization was categorized as urban and rural living area. The metabolic equivalent index (MET) was calculated by the codes and the detailed questions of the physical activity survey have been published elsewhere. [16-18] The same measurements of the health examination at baseline were taken after 18-year follow-up. 
All measurements of WC and BMI were collected according to the World Health Organization standard.[1920] WC was measured with gentle breathing at the midpoint between the lowest rib and the iliac crest to the nearest $0.1 \mathrm{~cm}$. The difference value (D-value) in WC was calculated by that the WC measured at baseline subtracted that measured at follow-up. Percent of WC gain $₫ 0 \%$ indicated that WC increased from baseline to follow-up, the greater the percent WC gain, the greater the increase in WC. Weight and height were measured with participants in light clothing and without shoes. The measurements of weight and height corrected to the nearest $0.5 \mathrm{~kg}$ and $0.1 \mathrm{~cm}$, respectively. Body mass index (BMI) was calculated as the ratio of weight $(\mathrm{kg})$ to the square of height $\left(\mathrm{m}^{2}\right)$.

Lung function was measured by respiratory physician using the same spirometer (MasterScreen Pneumo, Jaeger, Germany) and all examinations were in accordance with American Thoracic Society recommendation.[21] Firstly, all participants were led through practice the exhalations before the examination of lung function. Then, pre- and post-bronchodilator forced expiratory volume in one second $\left(\mathrm{FEV}_{1}\right)$ and forced vital capacity $(\mathrm{FVC})$ was measured as the primary outcomes and the ratio of these two measurements $\left(\mathrm{FEV}_{1}\right.$ : FVC) was calculated for each subject to assess the risk of COPD. Participants were asked to return on another day for an additional spirometry test, when the result of examination was regarded as low quality.

Overnight fasting blood was collected into vacuum tubes for assessing fasting plasma glucose (FPG), total cholesterol (TC), triglyceride (TG) and high-density lipoprotein cholesterol (HDL-C). Details about the storage and measurements methods were in accordance with the Clinical Practices standards.[22]

\section{Definitions}

Abdominal obesity for Chinese adults was defined by $W C \geq 90 \mathrm{~cm}$ for men and $W C \geq 80 \mathrm{~cm}$ for women according to the criteria of the Working Group on Obesity in China.[23]

According to the Global Initiative for Chronic Obstructive Lung Disease (GOLD) criteria, COPD was defined as a post-bronchodilator FEV1:FVC $<70 \%$.[24] Combined with the diagnose criteria for COPD in China, we also considered patients' history of illness, results of X-ray and biochemical examination when participants were diagnosed as COPD.[25] Patients of COPD were defined as a participant who reported a previous diagnosis of COPD through spirometry testing by physician. Participants were also defined as COPD patients by self-reporting that they received any inhaled short-acting or long-acting bronchodilator or corticosteroid therapy before.

\section{Statistical analysis}

All continuous variables were presented as mean (Standard Deviation) and categorical data as number (percentage). Kruskal-Wallis test or chi-square test was used to estimate the differences of variables in each group at baseline. To examine the association of WC dynamic change and incident COPD risk in detail, the range of percent WC change was divided into small categories: $\leq-2.5 \%,-2.5-2.5 \%, 2.5-5 \%$ and $>5 \%$. 
The three Poisson regression models were used to assess the association of the risk of COPD and WC gain by relative risk $(R R)$ and $95 \%$ confidence interval $(C l)$.[26-27] For the first part analysis, WC gain - 2.5-2.5\% was regarded as the reference group. Participants with normal WC at both baseline and follow-up were regarded as the reference group in the second part analysis. All potential confounding factors in the regression models were adjusted based on the baseline variables. Model 1 adjusted for age firstly. Then, model 2 adjusted for age as well as education level, smoking status, alcohol drinking, and physical activity. Finally, model 3 adjusted for all variables in model 2 as well as body mass index, systolic blood pressure (SBP), diastolic blood pressure (DBP), fasting plasma glucose (FPG), total cholesterol (TC), triglycerides (TG), high density lipoprotein cholesterol (HDL-C), forced expiratory volume in 1 second $\left(F E V_{1}\right)$ and forced vital capacity (FVC).

All analyses were performed by Stata, 12.0 version (Stata Corporation, College Station, TX, USA). A twotailed $p$ value less than 0.05 was regarded significantly.

\section{Results}

A total of 8,164 participants were included in this study. Baseline characteristics of the study population by WC gain category are in Table 1. Mean (SD) age for men and women was 27.5 (8.12) years and 27.3 (8.01) years, and mean (SD) WC at baseline was $82.3(9.56) \mathrm{cm}$ and $72.4(9.44) \mathrm{cm}$, respectively. During a median follow-up of 18.4 years, a total of 917 COPD cases (516 men) were identified. 
Table 1

Baseline characteristics of study participants stratified by percent of waist circumference (WC) change from baseline to follow-up.

\begin{tabular}{|c|c|c|c|c|c|}
\hline \multirow[b]{2}{*}{ Baseline } & \multicolumn{5}{|c|}{ Percent of WC gain (\%) } \\
\hline & $\leq-2.5$ & -2.5 to 2.5 & 2.5 to 5 & $\mathbf{8 5}$ & $\begin{array}{l}\mathrm{p}- \\
\text { value }\end{array}$ \\
\hline $\operatorname{Men}(n=3929)$ & 782 & 1190 & 570 & 1387 & \\
\hline Age(years) & $27.7(9.02)$ & $27.9(8.62)$ & $27.2(8.34)$ & $27.4(8.71)$ & 0.142 \\
\hline High educational (\%) & $128(16.4)$ & $226(19.0)$ & $97(17.1)$ & 221(15.9) & 0.201 \\
\hline Smoking (\%) & $558(71.4)$ & $852(71.6)$ & $412(72.3)$ & $986(71.1)$ & 0.961 \\
\hline Alcohol drinking (\%) & $238(30.4)$ & $361(30.3)$ & 178(31.2) & $456(32.9)$ & 0.498 \\
\hline $\begin{array}{l}\text { Physical activity (MET/hours per } \\
\text { week) }\end{array}$ & $285(25.8)$ & $278(27.9)$ & $257(28.9)$ & 255(29.7) & $\llbracket 0.001$ \\
\hline Urbanization & $267(34.2)$ & $410(34.5)$ & 195(34.2) & $485(35.0)$ & 0.978 \\
\hline $\mathrm{BMI}(\mathrm{kg} / \mathrm{m} 2)$ & $23.0(3.36)$ & 23.2(3.24) & $23.4(3.11)$ & $23.6(2.88)$ & 0.067 \\
\hline WC(cm) & $85.1(10.5)$ & $83.9(9.80)$ & $82.8(9.44)$ & $81.9(8.65)$ & 0.044 \\
\hline $\mathrm{SBP}(\mathrm{mm} \mathrm{Hg})$ & $118.8(11.8)$ & $118.3(11.7)$ & $118.1(10.4)$ & $117.2(10.5)$ & 0.105 \\
\hline $\mathrm{DBP}(\mathrm{mm} \mathrm{Hg})$ & 76.2(7.68) & $74.8(7.80)$ & $74.4(7.32)$ & $73.7(7.67)$ & 0.041 \\
\hline $\mathrm{FPG}(\mathrm{mg} / \mathrm{dl})$ & $89.5(14.4)$ & $91.2(20.1)$ & $90.4(17.8)$ & $92.3(18.2)$ & 0.079 \\
\hline $\mathrm{TC}(\mathrm{mmol} / \mathrm{L})$ & $4.38(0.87)$ & $4.38(0.91)$ & $4.36(0.88)$ & $4.22(0.91)$ & 0.008 \\
\hline $\mathrm{TG}(\mathrm{mmol} / \mathrm{L})$ & $1.77(1.32)$ & $1.63(1.12)$ & $1.64(1.18)$ & $1.68(0.89)$ & 0.101 \\
\hline $\mathrm{HDL}-\mathrm{C}(\mathrm{mmol} / \mathrm{L})$ & $1.16(0.26)$ & $1.10(0.28)$ & $1.11(0.25)$ & $1.13(0.27)$ & 0.112 \\
\hline $\mathrm{FEV}_{1}(\mathrm{~L})$ & $3.21(0.50)$ & $3.23(0.54)$ & $3.20(0.61)$ & $3.19(0.70)$ & 0.282 \\
\hline $\mathrm{FVC}(\mathrm{L})$ & $3.13(0.43)$ & $3.16(0.41)$ & $3.11(0.54)$ & $3.18(0.37)$ & 0.173 \\
\hline $\mathrm{FEV}_{1} / \mathrm{FVC}(\%)$ & 93.4 & 92.2 & 90.6 & 91.3 & \\
\hline Women $(n=4235)$ & 860 & 1058 & 567 & 1750 & \\
\hline Age(years) & $27.3(8.23)$ & $27.3(8.45)$ & $27.8(8.61)$ & $27.0(8.98)$ & 0.134 \\
\hline High educational (\%) & 182(21.2) & 198(18.7) & 113(19.9) & $301(17.2)$ & 0.087 \\
\hline Smoking (\%) & $3(0.35)$ & $2(0.19)$ & $1(0.18)$ & $2(0.11)$ & 0.640 \\
\hline $\begin{array}{l}\text { BMI, body mass index; WC, wais } \\
\text { pressure; } F P G \text {, fasting plasma gl } \\
\text { lipoprotein cholesterol; } F E V_{1} \text {, for } \\
\text { FEV }_{1} / F V C \text {, the ratio of forced exp }\end{array}$ & $\begin{array}{l}\text { umference; } \\
\text { e; TC, total } \\
\text { xpiratory vo } \\
\text { ry volume }\end{array}$ & $\begin{array}{l}\text { systolic bl } \\
\text { esterol; TG, } \\
\text { e in one se } \\
\text { e second a }\end{array}$ & $\begin{array}{l}\text { d pressure; } \\
\text { glyceride; } H \\
\text { nd; FVC, forc } \\
\text { forced vital }\end{array}$ & $\begin{array}{l}\text { diastolic bloc } \\
\text { C, high-density } \\
\text { vital capacity } \\
\text { pacity. }\end{array}$ & \\
\hline
\end{tabular}




\begin{tabular}{|c|c|c|c|c|c|}
\hline \multirow[b]{2}{*}{ Alcohol drinking (\%) } & \multicolumn{5}{|c|}{ Percent of WC gain (\%) } \\
\hline & $4(0.47)$ & $6(0.57)$ & $4(0.71)$ & $4(0.23)$ & 0.364 \\
\hline $\begin{array}{l}\text { Physical activity (MET/hours per } \\
\text { week) }\end{array}$ & $268(24.8)$ & $265(28.7)$ & $243(25.6)$ & $246(26.4)$ & $\varangle 0.001$ \\
\hline Urbanization & $299(34.8)$ & $364(34.4)$ & $200(35.2)$ & $600(34.3)$ & 0.975 \\
\hline $\mathrm{BMI}\left(\mathrm{kg} / \mathrm{m}^{2}\right)$ & $22.5(3.54)$ & 22.3(3.32) & $22.2(3.31)$ & $22.6(3.32)$ & 0.328 \\
\hline $\mathrm{WC}(\mathrm{cm})$ & $74.2(9.67)$ & $71.8(9.56)$ & $70.4(8.90)$ & 68.3(8.89) & $\varangle 0.001$ \\
\hline $\mathrm{SBP}(\mathrm{mm} \mathrm{Hg})$ & $116.5(11.3)$ & 115.7(11.7) & $114.3(11.6)$ & $115.8(11.5)$ & 0.099 \\
\hline $\mathrm{DBP}(\mathrm{mm} \mathrm{Hg})$ & $74.2(7.45)$ & $74.2(7.55)$ & 73.7(7.69) & $74.1(7.32)$ & 0.110 \\
\hline $\mathrm{FPG}(\mathrm{mg} / \mathrm{dl})$ & $89.8(14.7)$ & $90.8(17.6)$ & $91.4(18.6)$ & $91.1(18.0)$ & 0.073 \\
\hline $\mathrm{TC}(\mathrm{mmol} / \mathrm{L})$ & $4.44(0.99)$ & $4.49(0.97)$ & $4.44(0.97)$ & $4.41(0.87)$ & 0.093 \\
\hline $\mathrm{TG}(\mathrm{mmol} / \mathrm{L})$ & $1.65(1.13)$ & $1.69(1.12)$ & $1.67(1.06)$ & $1.68(0.98)$ & 0.236 \\
\hline $\mathrm{HDL}-\mathrm{C}(\mathrm{mmol} / \mathrm{L})$ & $1.21(0.27)$ & $1.19(0.27)$ & $1.23(0.29)$ & $1.22(0.26)$ & 0.082 \\
\hline $\mathrm{FEV}_{1}(\mathrm{~L})$ & $2.30(0.44)$ & $2.29(0.48)$ & $2.26(0.52)$ & $2.28(0.61)$ & 0.249 \\
\hline $\mathrm{FVC}(\mathrm{L})$ & $2.51(0.47)$ & $2.56(0.45)$ & $2.59(0.51)$ & $2.55(0.48)$ & 0.190 \\
\hline $\mathrm{FEV}_{1} / \mathrm{FVC}(\%)$ & 90.4 & 91.6 & 90.6 & 91.9 & \\
\hline \multicolumn{6}{|c|}{$\begin{array}{l}\text { BMI, body mass index; WC, waist circumference; } S B P \text {, systolic blood pressure; DBP, diastolic blood } \\
\text { pressure; FPG, fasting plasma glucose; TC, total cholesterol; TG, triglyceride; HDL-C, high-density } \\
\text { lipoprotein cholesterol; FEV } \text { F }_{1} \text {, forced expiratory volume in one second; FVC, forced vital capacity; }\end{array}$} \\
\hline
\end{tabular}

Our study investigated the COPD risk according to the WC dynamic gain. As shown in Table 2 and Table 3, the RRs for COPD increased significantly with rising percent WC gain among both male and female participants ( $p$ for trend $<0.01$ ). Compared with the group of $-2.5 \%$ to $2.5 \%$ WC change, the adjusted RR ( $95 \%$ Cls) for COPD with WC gain $₫ 5 \%$ was $1.19(95 \% C l=1.04-1.48)$ among men and $1.14(95 \% C l=1.01-1.40)$ among women. The COPD risk significantly decreased among men with WC loss over $2.5 \%(R R=0.82$, $95 \% \mathrm{Cl}=0.67-0.99)$. (Table 2 model 3 ) 
Table 2

Risk of chronic obstructive lung disease (COPD) by percent of waist circumference (WC) change for men

\begin{tabular}{|c|c|c|c|c|c|c|}
\hline \multirow{2}{*}{$\begin{array}{l}\text { Percent of WC gain } \\
\text { (\%) }\end{array}$} & \multirow[t]{2}{*}{ Total } & \multirow{2}{*}{$\begin{array}{l}\text { COPD } \\
\text { cases }\end{array}$} & \multirow[t]{2}{*}{ Incidence(\%) } & \multicolumn{3}{|c|}{$\mathrm{RR}(95 \% \mathrm{Cl})$} \\
\hline & & & & Model $1^{\mathrm{a}}$ & Model $2^{b}$ & Model $3^{c}$ \\
\hline \multicolumn{7}{|l|}{ Total } \\
\hline$\leq-2.5$ & 782 & 85 & 10.9 & $\begin{array}{l}0.85(0.66 \\
1.04)\end{array}$ & $\begin{array}{l}0.81(0.66 \\
0.99)\end{array}$ & $\begin{array}{l}0.82(0.67 \\
0.99)\end{array}$ \\
\hline-2.5 to 2.5 & 1190 & 152 & 12.8 & reference & reference & reference \\
\hline 2.5 to 5 & 570 & 75 & 13.1 & $\begin{array}{l}1.07(0.89 \\
1.30)\end{array}$ & $\begin{array}{l}0.98(0.79 \\
1.21)\end{array}$ & $\begin{array}{l}0.99(0.81 \\
1.21)\end{array}$ \\
\hline$\otimes 5$ & 1387 & 204 & 14.7 & $\begin{array}{l}1.11(0.91, \\
1.35)\end{array}$ & $\begin{array}{l}\text { 1.13(0.93, } \\
1.39)\end{array}$ & $\begin{array}{l}1.19(1.04, \\
1.48)\end{array}$ \\
\hline$P$ for trend & & & & $<0.001$ & $<0.001$ & $<0.001$ \\
\hline \multicolumn{7}{|c|}{ Non-abdominal obesity at baseline } \\
\hline$\leq-2.5$ & 511 & 43 & 8.4 & $\begin{array}{l}0.81(0.64 \\
1.02)\end{array}$ & $\begin{array}{l}0.73(0.55 \\
0.96)\end{array}$ & $\begin{array}{l}0.78(0.60 \\
1.01)\end{array}$ \\
\hline-2.5 to 2.5 & 885 & 94 & 10.6 & reference & reference & reference \\
\hline 2.5 to 5 & 451 & 51 & 11.3 & $\begin{array}{l}1.10(0.87 \\
1.39)\end{array}$ & $\begin{array}{l}0.99(0.77 \\
1.29)\end{array}$ & $\begin{array}{l}0.96(0.75 \\
1.22)\end{array}$ \\
\hline$\otimes 5$ & 1252 & 159 & 12.7 & $\begin{array}{l}1.19(1.02, \\
1.36)\end{array}$ & $\begin{array}{l}1.15(0.98 \\
1.35)\end{array}$ & $\begin{array}{l}1.15(1.06 \\
1.38)\end{array}$ \\
\hline$P$ for trend & & & & $<0.001$ & $<0.001$ & $<0.001$ \\
\hline \multicolumn{7}{|c|}{ Abdominal obesity at baseline } \\
\hline$\leq-2.5$ & 271 & 42 & 15.4 & $\begin{array}{l}0.85(0.64 \\
1.13)\end{array}$ & $\begin{array}{l}0.81(0.59 \\
1.10)\end{array}$ & $\begin{array}{l}0.86(0.65 \\
1.14)\end{array}$ \\
\hline-2.5 to 2.5 & 305 & 58 & 19.0 & reference & reference & reference \\
\hline 2.5 to 5 & 119 & 24 & 20.2 & $\begin{array}{l}1.05(0.75 \\
1.48)\end{array}$ & $\begin{array}{l}1.04(0.73, \\
1.50)\end{array}$ & $\begin{array}{l}1.13(0.80 \\
1.61)\end{array}$ \\
\hline
\end{tabular}

Data are relative risk (RR) and $95 \%$ Cls.

${ }^{a}$ Adjusted for age at baseline.

${ }^{\mathrm{b}}$ Adjusted for variables in model 1 as well as education level, smoking status, alcohol drinking, and physical activity at baseline.

${ }^{\mathrm{c}}$ Adjusted for variables in model 2 as well as body mass index, systolic blood pressure, diastolic blood pressure, fasting plasma glucose, total cholesterol, triglycerides, high density lipoprotein cholesterol, forced expiratory volume in one second and forced vital capacity at baseline. 


\begin{tabular}{|c|c|c|c|c|c|c|}
\hline \multirow{2}{*}{$\begin{array}{l}\text { Percent of WC gain } \\
\text { (\%) }\end{array}$} & \multirow[t]{2}{*}{ Total } & \multirow{2}{*}{$\begin{array}{l}\text { COPD } \\
\text { cases }\end{array}$} & \multirow[t]{2}{*}{ Incidence(\%) } & \multicolumn{3}{|c|}{$\mathrm{RR}(95 \% \mathrm{Cl})$} \\
\hline & & & & Model $1^{\mathrm{a}}$ & Model $2^{b}$ & Model $3^{c}$ \\
\hline$\otimes 5$ & 135 & 45 & 33.3 & $\begin{array}{l}1.45(1.07 \\
1.94)\end{array}$ & $\begin{array}{l}1.43(1.03 \\
1.96)\end{array}$ & $\begin{array}{l}1.55(1.14 \\
2.12)\end{array}$ \\
\hline$P$ for trend & & & & $<0.001$ & $<0.001$ & $<0.001$ \\
\hline \multicolumn{7}{|c|}{ Data are relative risk (RR) and $95 \% \mathrm{Cls.}$} \\
\hline \multicolumn{7}{|c|}{ a Adjusted for age at baseline. } \\
\hline \multicolumn{7}{|c|}{$\begin{array}{l}\text { b Adjusted for variables in model } 1 \text { as well as education level, smoking status, alcohol drinking, and } \\
\text { physical activity at baseline. }\end{array}$} \\
\hline \multicolumn{7}{|c|}{$\begin{array}{l}{ }^{c} \text { Adjusted for variables in model } 2 \text { as well as body mass index, systolic blood pressure, diastolic blood } \\
\text { pressure, fasting plasma glucose, total cholesterol, triglycerides, high density lipoprotein cholesterol, } \\
\text { forced expiratory volume in one second and forced vital capacity at baseline. }\end{array}$} \\
\hline
\end{tabular}


Table 3

Risk of chronic obstructive lung disease (COPD) by percent of waist circumference (WC) change for women

\begin{tabular}{|c|c|c|c|c|c|c|}
\hline \multirow{2}{*}{$\begin{array}{l}\text { Percent of WC } \\
\text { change (\%) }\end{array}$} & \multirow[t]{2}{*}{ Total } & \multirow{2}{*}{$\begin{array}{l}\text { COPD } \\
\text { cases }\end{array}$} & \multirow[t]{2}{*}{ Incidence(\%) } & \multicolumn{3}{|l|}{$\mathrm{RR}(95 \% \mathrm{Cl})$} \\
\hline & & & & Model 1 a & Model $2^{b}$ & Model $3^{c}$ \\
\hline \multicolumn{7}{|l|}{ Total } \\
\hline$\leq-2.5$ & 860 & 74 & 8.6 & $\begin{array}{l}0.88(0.75 \\
1.02)\end{array}$ & $\begin{array}{l}0.98(0.81 \\
1.19)\end{array}$ & $\begin{array}{l}0.98(0.83 \\
1.19)\end{array}$ \\
\hline-2.5 to 2.5 & 1058 & 110 & 10.4 & reference & reference & reference \\
\hline 2.5 to 5 & 567 & 44 & 7.8 & $\begin{array}{l}0.94(0.79 \\
1.11)\end{array}$ & $\begin{array}{l}1.03(0.84 \\
1.28)\end{array}$ & $\begin{array}{l}1.06(0.85 \\
1.30)\end{array}$ \\
\hline$\otimes 5$ & 1750 & 173 & 9.9 & $\begin{array}{l}1.07(0.95 \\
1.20)\end{array}$ & $\begin{array}{l}1.12(0.96 \\
1.31)\end{array}$ & $\begin{array}{l}1.14(1.01 \\
1.40)\end{array}$ \\
\hline$P$ for trend & & & & 0.005 & 0.007 & $<0.001$ \\
\hline \multicolumn{7}{|c|}{ Non-abdominal obesity at baseline } \\
\hline$\leq-2.5$ & 292 & 10 & 3.4 & $\begin{array}{l}0.87(0.66 \\
1.17)\end{array}$ & $\begin{array}{l}0.90(0.62 \\
1.20)\end{array}$ & $\begin{array}{l}0.94(0.65 \\
1.34)\end{array}$ \\
\hline-2.5 to 2.5 & 471 & 22 & 4.7 & reference & reference & reference \\
\hline 2.5 to 5 & 277 & 5 & 1.8 & $\begin{array}{l}0.78(0.56 \\
1.08)\end{array}$ & $\begin{array}{l}0.83(0.56 \\
1.23)\end{array}$ & $\begin{array}{l}0.92(0.61, \\
1.35)\end{array}$ \\
\hline$\nabla 5$ & 1045 & 49 & 4.7 & $\begin{array}{l}1.02(0.81 \\
1.29)\end{array}$ & $\begin{array}{l}1.04(0.79 \\
1.38)\end{array}$ & $\begin{array}{l}\text { 1.03(0.76, } \\
1.39)\end{array}$ \\
\hline$P$ for trend & & & & $<0.001$ & 0.008 & $<0.001$ \\
\hline \multicolumn{7}{|c|}{ Abdominal obesity at baseline } \\
\hline$\leq-2.5$ & 568 & 64 & 11.3 & $\begin{array}{l}0.82(0.69 \\
0.98)\end{array}$ & $\begin{array}{l}0.95(0.77 \\
1.18)\end{array}$ & $\begin{array}{l}0.95(0.76 \\
1.20)\end{array}$ \\
\hline-2.5 to 2.5 & 587 & 88 & 14.9 & reference & reference & reference \\
\hline 2.5 to 5 & 290 & 39 & 13.4 & $\begin{array}{l}\text { 1.02(0.83, } \\
1.30)\end{array}$ & $\begin{array}{l}1.08(0.83 \\
1.42)\end{array}$ & $\begin{array}{l}1.10(0.82 \\
1.43)\end{array}$ \\
\hline
\end{tabular}

Data are relative risk (RR) and $95 \%$ Cls.

${ }^{\text {a }}$ Adjusted for age at baseline.

${ }^{\mathrm{b}}$ Adjusted for variables in model 1 as well as education level, smoking status, alcohol drinking, and physical activity at baseline.

${ }^{\mathrm{c}}$ Adjusted for variables in model 2 as well as body mass index, systolic blood pressure, diastolic blood pressure, fasting plasma glucose, total cholesterol, triglycerides, high density lipoprotein cholesterol, forced expiratory volume in one second and forced vital capacity at baseline. 


\begin{tabular}{|c|c|c|c|c|c|c|}
\hline \multirow{2}{*}{$\begin{array}{l}\text { Percent of WC } \\
\text { change (\%) }\end{array}$} & \multirow[t]{2}{*}{ Total } & \multirow{2}{*}{$\begin{array}{l}\text { COPD } \\
\text { cases }\end{array}$} & \multirow[t]{2}{*}{ Incidence(\%) } & \multicolumn{3}{|c|}{$\mathrm{RR}(95 \% \mathrm{Cl})$} \\
\hline & & & & Model $1^{\text {a }}$ & Model $2^{b}$ & Model $3^{c}$ \\
\hline$\nabla 5$ & 705 & 124 & 17.6 & $\begin{array}{l}1.28(1.05 \\
1.56)\end{array}$ & $\begin{array}{l}1.24(1.02, \\
1.52)\end{array}$ & $\begin{array}{l}1.27(1.01 \\
1.35)\end{array}$ \\
\hline$P$ for trend & & & & 0.009 & 0.004 & $<0.001$ \\
\hline \multicolumn{7}{|c|}{ Data are relative risk (RR) and $95 \% \mathrm{Cls.}$} \\
\hline \multicolumn{7}{|c|}{ a Adjusted for age at baseline. } \\
\hline \multicolumn{7}{|c|}{$\begin{array}{l}{ }^{\mathrm{b}} \text { Adjusted for variables in model } 1 \text { as well as education level, smoking status, alcohol drinking, and } \\
\text { physical activity at baseline. }\end{array}$} \\
\hline \multicolumn{7}{|c|}{$\begin{array}{l}{ }^{\mathrm{c}} \text { Adjusted for variables in model } 2 \text { as well as body mass index, systolic blood pressure, diastolic blood } \\
\text { pressure, fasting plasma glucose, total cholesterol, triglycerides, high density lipoprotein cholesterol, } \\
\text { forced expiratory volume in one second and forced vital capacity at baseline. }\end{array}$} \\
\hline
\end{tabular}

Moreover, participants were analyzed according to abdominal obesity or not at baseline. As shown in Table 2 and Table 3, the risk of COPD will increase 55\% $(R R=1.55,95 \% C l=1.14-2.12)$ and $27 \%(R R=1.27$, $95 \% C l=1.01-1.35)$ comparing to the group of $-2.5-2.5 \%$ WC gain in male and female participants with abdominal obesity at baseline, respectively.

The risk of incident COPD by transition in abdominal obesity status was also investigated in our study. As shown in Table 4, the risk of incident COPD was significantly greater for both male and female participants who were abdominal obesity at follow-up regardless of their abdominal obesity status at baseline comparing with participants who were not abdominal obesity at both baseline and follow-up. The risk of incident COPD increased $42 \%(R R=1.42,95 \% C l=1.05-1.85)$ for men and $22 \%(R R=1.22,95 \% C l=1.03-$ 1.68) for women with normal WC at baseline and abdominal obesity at follow-up. Additionally, the risk of new-onset COPD was more significant among male and female participants with abdominal obesity at both baseline and follow-up. 
Table 4

Risk of chronic obstructive lung disease (COPD) by abdominal obesity at baseline and follow-up

\begin{tabular}{|c|c|c|c|c|c|c|}
\hline \multirow{2}{*}{$\begin{array}{l}\text { Abdominal } \\
\text { obesity at } \\
\text { baseline }\end{array}$} & \multirow{2}{*}{$\begin{array}{l}\text { Abdominal } \\
\text { obesity at } \\
\text { follow-up }\end{array}$} & \multirow{2}{*}{$\begin{array}{l}\text { No. of } \\
\text { events/participants }\end{array}$} & \multirow[t]{2}{*}{ Incidence(\%) } & \multicolumn{3}{|l|}{$\mathrm{RR}(95 \% \mathrm{Cl})$} \\
\hline & & & & Model $1^{\text {a }}$ & Model $2^{b}$ & Model $3^{c}$ \\
\hline \multicolumn{7}{|l|}{ Men } \\
\hline No & No & $264 / 2614$ & 10.1 & reference & reference & reference \\
\hline Yes & No & $22 / 147$ & 14.9 & $\begin{array}{l}1.13(0.79 \\
1.60)\end{array}$ & $\begin{array}{l}1.16(0.78 \\
1.69)\end{array}$ & $\begin{array}{l}1.12(0.73, \\
1.58)\end{array}$ \\
\hline No & Yes & $90 / 485$ & 18.6 & $\begin{array}{l}1.41(1.07 \\
1.80)\end{array}$ & $\begin{array}{l}1.46(1.09 \\
1.88)\end{array}$ & $\begin{array}{l}1.42(1.05 \\
1.85)\end{array}$ \\
\hline Yes & Yes & $140 / 683$ & 20.5 & $\begin{array}{l}1.48(1.26 \\
1.78)\end{array}$ & $\begin{array}{l}1.52 \\
(1.29 \\
1.87)\end{array}$ & $\begin{array}{l}1.49(1.22, \\
1.82)\end{array}$ \\
\hline \multicolumn{7}{|l|}{ Women } \\
\hline No & No & $76 / 1362$ & 5.6 & reference & reference & reference \\
\hline Yes & No & $22 / 228$ & 9.6 & $\begin{array}{l}1.17(0.90 \\
1.52)\end{array}$ & $\begin{array}{l}1.31(0.95 \\
1.80)\end{array}$ & $\begin{array}{l}1.17(0.84, \\
1.64)\end{array}$ \\
\hline No & Yes & $78 / 723$ & 10.8 & $\begin{array}{l}1.22(1.02 \\
1.48)\end{array}$ & $\begin{array}{l}1.25(0.98 \\
1.59)\end{array}$ & $\begin{array}{l}1.22(1.03, \\
1.68)\end{array}$ \\
\hline Yes & Yes & $225 / 1922$ & 11.7 & $\begin{array}{l}1.35(1.07 \\
1.86)\end{array}$ & $\begin{array}{l}1.42(1.13, \\
1.89)\end{array}$ & $\begin{array}{l}1.36(1.04, \\
1.88)\end{array}$ \\
\hline \multicolumn{7}{|c|}{ Data are relative risk (RR) and $95 \% \mathrm{Cls.}$} \\
\hline \multicolumn{7}{|c|}{ Adjusted for age at baseline. } \\
\hline \multicolumn{7}{|c|}{$\begin{array}{l}\text { b Adjusted for variables in model } 1 \text { as well as education level, smoking status, alcohol drinking, and } \\
\text { physical activity at baseline. }\end{array}$} \\
\hline
\end{tabular}

Finally, our study re-estimated the risk of incident COPD with transition in abdominal obesity status for excluding the effect of smoking cigarettes on incident COPD. As shown in Table 5, the risk of COPD onset was basically consistent with the results for men $(R R=1.44,95 \% C l=1.01-2.21)$ and women $(R R=1.36$, $95 \% \mathrm{Cl}=1.04-1.92)$ in Table 4. The similar risk suggested an independent effect of WC on the incident COPD. 
Table 5

Sensitivity analysis of association between change of waist circumference (WC) and the risk of chronic obstructive lung disease (COPD) among participants who reported never smoking

\begin{tabular}{|c|c|c|c|c|c|c|}
\hline \multirow{2}{*}{$\begin{array}{l}\text { Abdominal } \\
\text { obesity at } \\
\text { baseline }\end{array}$} & \multirow{2}{*}{$\begin{array}{l}\text { Abdominal } \\
\text { obesity at } \\
\text { follow-up }\end{array}$} & \multirow{2}{*}{$\begin{array}{l}\text { No. of } \\
\text { events/participants }\end{array}$} & \multirow[t]{2}{*}{ Incidence(\%) } & \multicolumn{3}{|c|}{$\mathrm{RR}(95 \% \mathrm{Cl})$} \\
\hline & & & & Model $1^{a}$ & Model $2^{b}$ & Model $3^{c}$ \\
\hline \multicolumn{7}{|l|}{ Men } \\
\hline No & No & $70 / 732$ & 9.6 & reference & reference & reference \\
\hline Yes & No & $6 / 58$ & 10.3 & $\begin{array}{l}1.09(0.62 \\
1.90)\end{array}$ & $\begin{array}{l}1.12(0.55 \\
2.03)\end{array}$ & $\begin{array}{l}1.10 \\
(0.60 \\
1.98)\end{array}$ \\
\hline No & Yes & $26 / 134$ & 19.4 & $\begin{array}{l}1.43(0.95 \\
2.17)\end{array}$ & $\begin{array}{l}1.50(1.03 \\
2.28)\end{array}$ & $\begin{array}{l}1.44(1.01, \\
2.21)\end{array}$ \\
\hline Yes & Yes & $45 / 197$ & 22.8 & $\begin{array}{l}1.50(1.13 \\
2.19)\end{array}$ & $\begin{array}{l}1.58 \\
(1.19 \\
2.31)\end{array}$ & $\begin{array}{l}1.50 \\
(1.20 \\
2.28)\end{array}$ \\
\hline
\end{tabular}

Women

\begin{tabular}{lllllll} 
No & No & $76 / 1360$ & 5.6 & reference & reference & reference \\
Yes & No & $20 / 226$ & 8.8 & $\begin{array}{l}1.16(0.90, \\
1.57)\end{array}$ & $1.83)$ & $1.65)$ \\
\hline No & Yes & $78 / 720$ & 10.8 & $\begin{array}{l}1.22(1.02, \\
1.48)\end{array}$ & $1.25(0.98$, & $1.22(0.95$, \\
& & & & $1.59)$ & $1.55)$ \\
\hline Yes & Yes & $224 / 1921$ & 11.7 & $1.35(1.06$, & $1.42(1.13$, & $1.36(1.04$, \\
& & & & $1.87)$ & $1.88)$ & $1.92)$
\end{tabular}

Data are relative risk (RR) and $95 \%$ Cls.

${ }^{\text {a }}$ Adjusted for age at baseline.

${ }^{\mathrm{b}}$ Adjusted for variables in model 1 as well as education level, smoking status, alcohol drinking, and physical activity at baseline.

${ }^{c}$ Adjusted for variables in model 2 as well as body mass index, systolic blood pressure, diastolic blood pressure, fasting plasma glucose, total cholesterol, triglycerides, high density lipoprotein cholesterol, forced expiratory volume in one second and forced vital capacity at baseline.

\section{Discussion}

To our knowledge, this is the first long-term cohort study to investigate the association of WC dynamic change and the incidence of COPD among Chinese adults. This study showed that WC gain was significantly and positively associated with the risk of COPD in Chinese adult. Additionally, our study suggested a dose-response relationship between WC gain and incident COPD for both male and female 
participants regardless of abdominal obesity status at baseline after adjusting for relative covariates at baseline.

With the implementation of the opening and reforming policies since 1978, Chinese economy enter a rapid period of development.[28] According to the reports of National Bureau of Statistics, gross domestic product per capita increased from 381 Yuan in 1978 to 38,420 Yuan in 2012.[29] The patterns in changes of dietary and physical activity led the prevalence of obesity to a higher rate in China during past decades.[30] Available evidence showed that age-adjusted prevalence of abdominal obesity increased from $9.60-35.3 \%$ among men and from 30.5-51.7\% for women during 1993-2011 China Health and Nutrition Survey.[31] Furthermore, the age-adjusted prevalence of COPD defined by spirometry test in China increased rapidly from $8.2 \%$ in 2002-2004 to $13.7 \%$ in 2012-2015.[4] Unfortunately, the results of our study suggested abdominal obesity was closely associated with low level of $\mathrm{FEV}_{1}$ and $\mathrm{FVC}$ and the high risk of incident COPD. Hence, comprehensive strategies aiming at the prevention of abdominal obesity are urgently needed to reduce the increase in societal burden of lung diseases.

Few studies assessed the relationship between abdominal obesity and lung function and relative diseases. $[14-15,32]$ Pan et al. found that central adiposity and WC, but not general adiposity and BMI, was independently associated with lower pulmonary function and higher risk of restrictive respiratory defect in older Chinese.[32] The detail data showed participants with only central adiposity had lower pulmonary function comparing to those with neither central nor general obesity (adjusted $\beta$ range from -2.85 to -6.02 for $\mathrm{FEV}_{1} \%$ and $\mathrm{FVC} \%$, adjusted $\mathrm{OR}$ range from 1.14 to 1.70 , all $\mathrm{P}<0.05$ ). Another study conducted in Chinese children, which showed that waist-to-chest ratio (WCR) and WC was inversely associated with the lung function. The results showed that each 0.01 increase in WCR was associated with $8.14 \mathrm{ml}$ decrease for FVC, $9.36 \mathrm{ml}$ decrease for $\mathrm{FEV}_{1}$, and $6.54 \%$ decrease for $\mathrm{FEV}_{1} / \mathrm{FVC}$.[15] These results were consistent with our study that WC increase was positively associated with the low lung function and the incidence of COPD. On the contrary, Marga et al. found that large WC were not associated with $\mathrm{FEV}_{1}$ and FVC among 8-year-old children, but they suggested that this association may change over the course of life from childhood to adulthood.[14] However, all of these studies only focused on the association of WC and lung function in a cross-sectional level, which were lack of estimation on relationship of WC dynamic change and lung function and relative disease. Our study firstly used percent WC gain to investigate the lung function and the risk of incident COPD in Chinese population and found that both man and women with increased WC gain during 18-year follow-up shared a significantly increased risk of COPD after adjustment for important covariates.

Two potential mechanisms may give some explanations of the association of WC gain with the increase risk of COPD. On the one hand, WC gain can affect lung function through mechanical effects on lungs. Adiposity accumulation in the abdominal and thoracic regions may directly reduce vital capacity by limiting the room for lung expansion during inspiration and led to expiratory flow limitation finally.[33] These mechanisms are also likely to give out the explanation of the different risk of COPD between gender, which was consistent with previous studies,[34-35] because men tend to accumulate more fat mass in the abdominal area than women.[36] On the other hand, WC gain can damage lung tissue by inflammatory processes, and fat tissue is a source of inflammatory mediators that can impair lung function and decrease 
airway diameter.[37-38] The major cause of COPD was the drop of $\mathrm{FEV}_{1}$, which was closely associated with the lung inflammation related to high serum levels of C-reactive protein (CRP).[39] Abdominal obesity was the main factor which increased CRP concentrations resulted from adiposity fat accumulation.[40] Systemic inflammation might lead to reduced lung function given that CRP and interleukin-6 (IL-6) expressed in inflammatory in inflammatory lung epithelial cells.[41]

\section{Strengths And Limitations}

Our study has several strengths including the large sample size, long-term follow-up and prospective study design. Included participants were all free of COPD and other lung disease at baseline, which benefited to truly investigate the relationship between WC change and the risk of incident COPD.

There were also some limitations in this study. Firstly, the study participants were recruited from one city in the north of China, which may be lack of the representation of Chinese adults. However, there were more than $91 \%$ Han Chinese population in China, and participants in our study were mainly included the individuals with Han nationality (more than 93\%). Hence, the lifestyle and dietary factors were similar between persons. Then, the information of smoking, drinking and physical activity level was self-reported, which may misestimate the initiation rates due to the use of retrospective questions. Finally, although our study adjusted the relative covariates, some possible confounding was not available in this study.

\section{Conclusions}

In conclusion, this study provided the epidemiological evidence to better understand the effect of WC change on the risk of incident COPD. Change in WC is a significant predictor of incident COPD in Chinese adults. Staying a healthy lifestyle and regular physical activity are two important and common ways to keep away high risk of WC gain.

\section{Abbreviations}

WC

waist circumference; COPD:chronic obstructive pulmonary disease; FVC:forced vital capacity; FEV 1 :forced expiratory volume in $1 \mathrm{~s}$; MET:metabolic equivalent index; BMI:body mass index; SBP:systolic blood pressure; DBP:diastolic blood pressure; FPG:fasting plasma glucose; TC:total cholesterol; TG:triglycerides; HDL-C:high density lipoprotein cholesterol; RR:relative risk; Cl:confidence interval; SD:standard deviation; CRP:C-reactive protein; IL-6:interleukin-6.

\section{Declarations}

\section{Acknowledgement}

Thanks for the dedicated participants and all research staff of the study sincerely.

\section{Authors' contributions}


Ying-Hao Su contributed to study design, write the draft and revise the manuscript. Ma supported to collect and analyze the data. Han-Jun Zhao contributed to revise the manuscript.

\section{Funding}

This research did not receive any specific grant from funding agencies in the public, commercial, or not-forprofit sectors.

\section{Availability of data and materials}

The datasets used and/or analyzed during the current study are available from the corresponding author on reasonable request.

\section{Ethics approval and consent to participate}

The study protocol was approved by the ethics review committees of the Traditional Chinese Medicine Hospital of Hebei Province.

\section{Consent for publication}

Not applicable.

\section{Competing interests}

The authors declared no competing interests.

\section{References}

1. Adeloye D, Chua S, Lee C, Basquill C, Papana A, Theodoratou E, Nair H, Gasevic D, Sridhar D, Campbell H, Chan KY, Sheikh A, Rudan I, Global Health Epidemiology Reference Group (GHERG). Global and regional estimates of COPD prevalence: Systematic review and meta-analysis. J Glob Health. 2015;5:020415.

2. World Health Organization. Global surveillance, prevention and control of chronic respiratory diseases: a comprehensive approach. Geneva: WHO; 2018.

3. GBD 2016 Causes of Death Collaborators. Global, regional, and national age- sex specific mortality for 264 causes of death, 1980-2016: a systematic analysis for the Global Burden of Disease Study 2016. Lancet. 2017;390:1151-210.

4. Tang WenFang, Liu RiHui Yu, YaQin L, Jin G, Peng. Wang Ke.[Prevalence of COPD among Chinese People Aged 40 Years and Over From 2000 to 2014: A Meta-analysis]. J Jilin Univ. 2015;41(005):961-8.

5. World Health Organization. Risk factors for chronic respiratory diseases. In: Bousquet J, Khaltaev N. Global Surveillance, Prevention and Control of Chronic Respiratory Diseases: A Comprehensive Approach. Geneva. 2007:37-55.

6. Cai L, Wang XM, Fan LM, et al. Socioeconomic variations in chronic obstructive pulmonary disease prevalence, diagnosis, and treatment in rural Southwest China. BMC Public Health, 2020, 20(1). 
7. Siddharthan T, Grigsby MR, Goodman D, Chowdhury M, Rubinstein A, Irazola V, Laura G, Jaime Miranda J, Bernabe-Ortiz A, Alam D, Kirenga B, Jones R, van Gemert F, Wise RA, Checkley W. Association between household air pollution exposure and chronic obstructive pulmonary disease outcomes in 13 low- and middle-income country settings. Am J RespirCrit Care Med. 2018;197:611-20.

8. Salvi S, Barnes PJ. Is exposure to biomass smoke the biggest risk factor for COPD Globally Chest. 2010;138:3-6.

9. Jones RL, Nzekwu MM. eeects of body mass index on lung volumes. Chest. 2006;130:827-33.

10. Costa D, Barbalho MC, Miguel GPS, Forti EMP, Azevedo JL. M. C. Impact of Obesity on Pulmonary Function in Adult Women. Clinics. 2008;63:719-24.

11. Banerjee J, et al. Association of Body Mass Index (BMI) with Lung Function Parameters in Nonasthmatics Identified by Spirometric Protocols. Journal of Clinical Diagnostic Research: JCDR. 2014;8:12-4.

12. Iyer AS, Dransfield MT. The "Obesity Paradox" in Chronic Obstructive Pulmonary Disease: Can It Be Resolved? Ann Am ThoracSoc. 2018;15(2):158-9.

13. Wu TD, Mccormack MC, Wise RA, et al. The Obesity Paradox in COPD Is Absent in U.S. Never-Smokers. American Thoracic Society 2019 International Conference, May 17-22, 2019-Dallas, TX. 2019.

14. Bekkers MBM, Wijga AH, De Jongste JC, et al. Waist circumference, BMI, and lung function in 8-year-old children: The PIAMA birth cohort study. Pediatr Pulmonol. 2013;48(7):674-82.

15. Feng K, Chen LI, Han SM, et al. Ratio of waist circumference to chest circumference is inversely associated with lung function in Chinese children and adolescents. Respirology. 2012;17(7):1114-8.

16. Ng SW, Norton EC, Popkin BM. Why has physical activity levels declined among Chinese adults? Findings from the 1991-2006China health and nutrition surveys. Soc Sci Med. 2009;68:1305-14.

17. Ng SW, Popkin BM. Time use and physical activity: a shift away from movement across the globe. Obes Rev. 2012;13:659-80.

18. Ng SW, Howard AG, Wang HJ, Su C, Zhang B. The physical activity transition among adults in China: 1991-2011. Obes Rev. 2014;15(Suppl 1):27-36.

19. Geographical variation in. the major risk factors of coronary heart disease in menand women aged 3564 years. the WHO MONICA project. World Health Stat Q. 1988;41:115-40.

20. Zhou BF. Predictive values of body mass index and for risk factorsof certain related diseases in Chinese adults study on optimal cut offpoints of body mass index. Biom Env Sci. 2002;15:83-96.

21. American Thoracic Society. Standardization of spirometry, 1994 update. AmJ Respir Crit Care Med. 1995;152:1107-36.

22. Ash JS, Mccormack JL, Sittig DF, et al. Standard practices for computerized clinical decision support in community hospitals: a national survey. J Am Med Inform Assoc. 2012;19(6):980-7.

23. Alberti Kg, Zimmet P, shaw J. IDF epidemiology Task Force Consensus group. Themetabolic syndromea new worldwide definition. Lancet 2005;366:1059-62.

24. Global Initiative for Chronic Obstructive Lung Disease (GOLD). Global strategy for the diagnosis, management, and prevention of chronic obstructive pulmonary disease (2017 report) 2007. 
25. MarAR, Rodrigues DM, Dias M, et al. Classification of Chronic Obstructive Pulmonary Disease (COPD) according to the new Global Initiative for Chronic Obstructive Lung Disease (GOLD) 2017. Journal of Chronic Obstructive Pulmonary Disease, 2017:1-6.

26. Liu H. Robust Standard Error Estimate for Cluster Sampling Data: ASAS = IML Macro Procedure for Logistic Regression with Huberization.

27. Simcoe T. XTPQML: Stata module to estimate Fixed-effectsPoisson (Quasi-ML) regression with robust standard errors.Statistical Software Components 2007.

28. Yang G, Wang Y, Zeng Y R1990-2010: findings from the Global Burden of Disease Study 2010. Lancet, 2013; 381:1987-2015. https://doi.10.1016/S0140-6736(13)61097-1.apid health transition in China.

29. National Bureau of Statistics. http://www.stats.gov.cn/.

30. Wang h, Wang J, Liu MM, et al. Epidemiology of general obesity, abdominal obesity and related risk factors in urban adults from 33 communities of northeast China: the CHPSNE study. BMC Public Health. 2012;12:967.

31. The prevalence. and secular trends of abdominal obesity among Chinese adults, 1993-2011. Ann Epidemiol. 2015;25(10):797-9.

32. Pan J, Xu L, Lam TH, et al. Association of adiposity with pulmonary function in older Chinese: Guangzhou Biobank Cohort Study. Respir Med. 2017;132:102.

33. Salome CM, King GG, Berend N. Physiology of obesity and effects on lung function. J Appl Physiol. 2010;108(1):206.

34. Chinn S, Jarvis D, Melotti R, et al. Smoking cessation, lung function, and weight gain:a follow- up study. Lancet. 2005;365:1629-35.

35. Marcon A, Corsico A, Cazzoletti L, et al. Body mass index, weight gain, and other determinants of lung function decline in adult asthma. Journal of Allergy Clinical Immunology. 2009;123(5):1069-74.e4.

36. HarikKhan RI, Wise RA, Fleg JL. The effect of gender on the relationship between body fat distribution and lung function. J Clin Epidemiol. 2001;54(4):399-406.

37. JR AWF. Obesity-induced inflammation: a metabolic dialogue in the language of inflammation. J Intern Med. 2007;262(4):408-14.

38. Hancox RJ, Poulton R, Greene JM, et al. Systemic inflammation and lung function in young adults. Thorax. 2007;62(12):1064.

39. Eid AA, et al. Inflammatory response and body composition in chronic obstructive pulmonary disease. American journal of respiratory and critical care medicine 164, 1414-1418.

40. Faber DR, Van der Graaf Y, Westerink J, Visseren FL. Increased visceral adipose tissue mass is associated with increased C-reactive protein in patients with manifest vascular diseases. Atherosclerosis 212, 274-280.

41. Gould JM, Weiser JN. Expression of C-reactive protein in the Human Respiratory Tract. Infection and Immunity 69, 1747-1754. 